\title{
REVUE SEMESTRIELLE DES PUBLICATIONS MATHÉMATIQUES
}

VEREENIGD MET

\section{JAHRBUCH ÜBER DIE}

FORTSCHRITTE DER MATHEMATIK

UITGEGEVEN DOOR DE

PREUSSISCHE AKADEMIE DER WISSENSCHAFTEN

MET BIJZONDERE MEDEWERKING VAN HET

WISKUNDIG GENOOTSCHAP TE AMTSERDAM

REDACTIE GEORG FEIGL, HENDRIK DE VRIES

REVUE SEMESTRIELLE DES

PUBLICATIONS MATHÉMATIQUES

DEEL 37. JAARGANG 1932. NR. 4

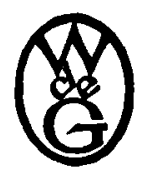

W A L T E R D E G R U Y T E R \& C O. VORMALS G.J. GÖSCHEN'SCHE VERLAGSHANDLUNG - J. GUTTENTAG, VERLAGSBUCHHANDLUNG - GEORG REIMER - KARL J. TRÜBNER - VEIT \& COMP. $\begin{array}{lll}\text { BERLIN LEIPZIG } & 1932 \quad \text { LE }\end{array}$ 